\title{
Multipactoring suppression by fine grooving of conductor surfaces of coaxial-line input couplers for high beam current storage rings
}

\author{
Tetsuo Abe, ${ }^{*}$ Tatsuya Kageyama, Hiroshi Sakai, Yasunao Takeuchi, and Kazuo Yoshino \\ Accelerator Laboratory, High Energy Accelerator Research Organization (KEK), Tsukuba, Ibaraki 305-0801, Japan
}

(Received 9 February 2010; published 4 October 2010)

\begin{abstract}
Input couplers for radio-frequency accelerating cavities with heavy beam loading undergo many multipactoring zones due to the wide range of input powers. Furthermore, regular coaxial lines have more multipactoring than rectangular waveguides because of more uniformity of the electromagnetic field. Grooving conductor surfaces of coaxial lines is expected to be a promising method for suppressing multipactoring under any conditions with heavy beam loading. In this paper, we present a method for designing practical fine grooves on conductor surfaces of coaxial lines to suppress multipactoring, based on multipactoring zone maps, and demonstrate the suppressive power both in high-power tests and accelerator operations.
\end{abstract}

DOI: 10.1103/PhysRevSTAB.13.102001

PACS numbers: 29.20.db

\section{INTRODUCTION}

Radio-frequency (rf) input couplers (couplers) are one of the most important components in high-power rf systems. In the normal-conducting accelerating cavity system ARES $^{1}$ [1-3] for the KEK B-factory (KEKB) [3-12], coaxial-line couplers are used to feed high power from klystrons into accelerating cavities with heavy beam loading. Figure 1 shows a schematic diagram of the coupler for the ARES, where the coaxial line, which is made of oxygen-free copper and has an outer (inner) radius of the inner (outer) conductor of $16.7 \mathrm{~mm}(38.5 \mathrm{~mm})$, has a loop at its end for coupling to the magnetic field of the $\mathrm{TE}_{013}$ mode in the energy-storage cavity of the three-cavity system of the ARES [13]. The couplers for the ARES have been tested in a test stand for time-averaged powers of the continuous wave (CW) of up to and over $800 \mathrm{~kW}[14,15]$.

The couplers, which had passed high-power tests, have been used in KEKB operations, where 20 accelerating cavities of the ARES (ARES cavities) are used to store a $3.5-\mathrm{GeV}$ positron beam in the low-energy ring (LER), and 12 ARES cavities together with eight superconducting cavities are used to store an $8.0-\mathrm{GeV}$ electron beam in the high-energy ring (HER). The design values of the accelerating voltage and the wall-loss power of the ARES are $0.5 \mathrm{MV}$ and $150 \mathrm{~kW}$, respectively, and the normal operating voltage range is 0.3 to $0.4 \mathrm{MV}$. Operation of the ARES cavities has generally been stable with low trip rates (typically less than one trip per cavity for three months of operation), so that the ARES is one of the key components which enable KEKB to store high beam currents of about 1.6 $\mathrm{A}$ in the LER and about $1.2 \mathrm{~A}$ in the HER, and to produce the world's highest luminosities.

\footnotetext{
*tetsuo.abe@kek.jp

${ }^{1}$ ARES stands for accelerator resonantly coupled with energy storage.
}

In 2003 and 2004, we had a serious problem caused by multipactoring [16] in the coaxial lines of the couplers for two of the 32 ARES cavities used in KEKB operations. Multipactoring phenomena can be experimentally detected as vacuum pressure rises in the cavities, temperature rises in the cooling water from the couplers, and discharges observed using TV cameras through the inside of the cavities, where all of these should be dependent on the input rf power. There was no conditioning effect and no improvement in one of the two cavities even after the couplers had been replaced by new ones which had passed high-power tests without exhibiting multipactoring symptoms and the inner surfaces of the coupler ports had been scraped and wiped to remove the possibility of multipactoring sources in the cavities. This troublesome cavity, referred to as CavityM [17] in [18], unquestionably has some multipactoring sources inside. It should be mentioned that a water leak into the vacuum, which occurred

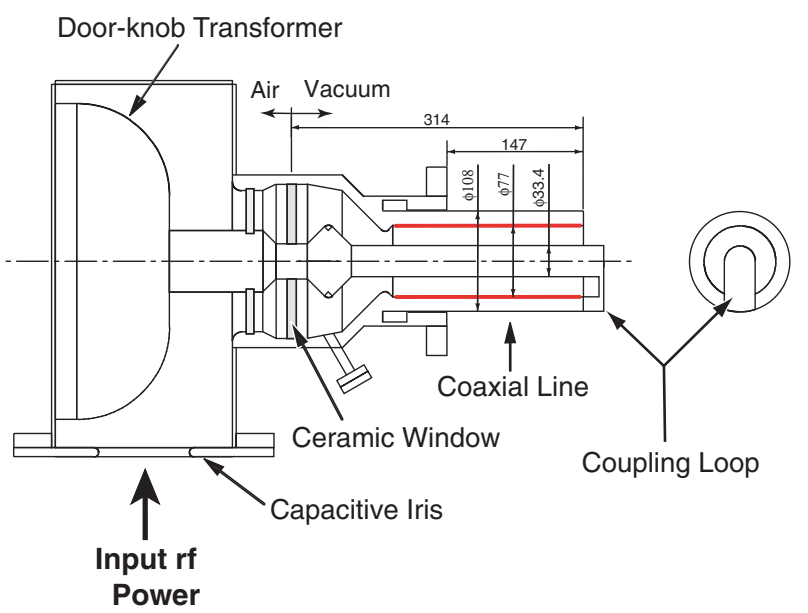

FIG. 1. Schematic diagram of the input coupler for the ARES. The red lines indicate the region where fine grooving is to be applied. The dimensions are expressed in units of millimeters. 


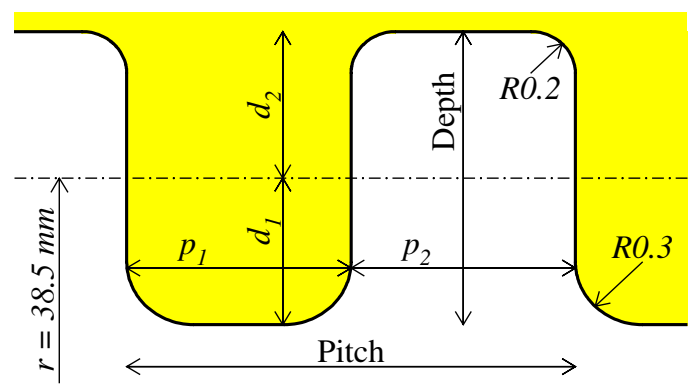

FIG. 2. Definitions of pitch and depth in this paper. The solid line indicates a grooved surface of the outer conductor (yellow area) of the coaxial line. The dash-dotted line indicates the radial position of the inner surface of the ungrooved outer conductor: $r=38.5 \mathrm{~mm}$. We assume that $p_{1}=p_{2}=$ pitch $/ 2$ and $d_{1}=$ $d_{2}=$ depth $/ 2$ in this groove design.

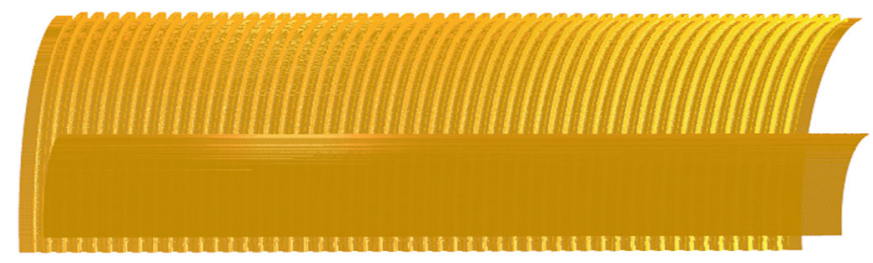

FIG. 3. An example of the geometries for grooved coaxial lines used in this simulation by GDFIDL. This shows the case for a pitch of $2.0 \mathrm{~mm}$ and a depth of $1.4 \mathrm{~mm}$.

in 2001 near CavityM, is a possible origin for the production of multipactoring sources, and that there have been no water leaks into the vacuum near any of the other ARES cavities. From a point of view that multipactoring-free couplers should be developed toward a higher luminosity B-factory, where the vacuum pressures in the ARES cavities will be higher due to the higher beam currents, we have made a decision not to replace CavityM, but rather to use it as a test stand at the KEKB accelerator.

The above-mentioned multipactoring problem has been temporarily solved without replacing the whole cavity system of CavityM by operating the cavity in a multipactoring-free region in the multipactoring zone map [18]. However, this method limits the input rf power to the cavity $\left(P_{\text {in }}\right)$. As a more permanent solution, we have developed a promising method by fine grooving of conductor surfaces of coaxial lines. Grooved surfaces have been expected to substantially suppress multipactoring (e.g., see $[19,20]$ ), where its suppressive power is, in principle, independent of the condition of the conductor surface, such as secondary electron yield. Finally, we have demonstrated the performance of couplers with fine grooves (grooved couplers) not only in a test stand but also in KEKB operations.

\section{MULTIPACTORING SIMULATION}

The simulation method used in this paper is based on the one described in [18], where cylindrical coordinates $(r, \theta, z)$ are used, and electron motion is calculated by numerically solving the relativistic equation of motion. In the following Secs. II A, II B, and II C, we describe the differences from and the additions to the definitions and calculations in the simulation method used in [18]. In Sec. II D, we determine the groove design.

\section{A. Electromagnetic field of the quasi-TEM}

The $z$ axis is chosen to lie along the axis of the coaxial line with the outer radius of the inner conductor: $16.7 \mathrm{~mm}$. Fine grooving is applied to the region of $-50 \mathrm{~mm}<z<$ $50 \mathrm{~mm}$ in the azimuthal direction, but only to the inner surface of the outer conductor because multipactoring is almost single sided on the outer conductor in the case of the ARES. We define "pitch" and "depth" of the grooving as shown in Fig. 2, and blend the edges and corners with blend radii of 0.3 and $0.2 \mathrm{~mm}$, respectively. Here we assume that the outermost radii of the inner surface of the outer conductor are equal to $r=38.5 \mathrm{~mm}+$ depth $/ 2$, the innermost radii of the inner surface of the outer conductor are equal to $r=38.5 \mathrm{~mm}-$ depth $/ 2$, and the widths of the concave
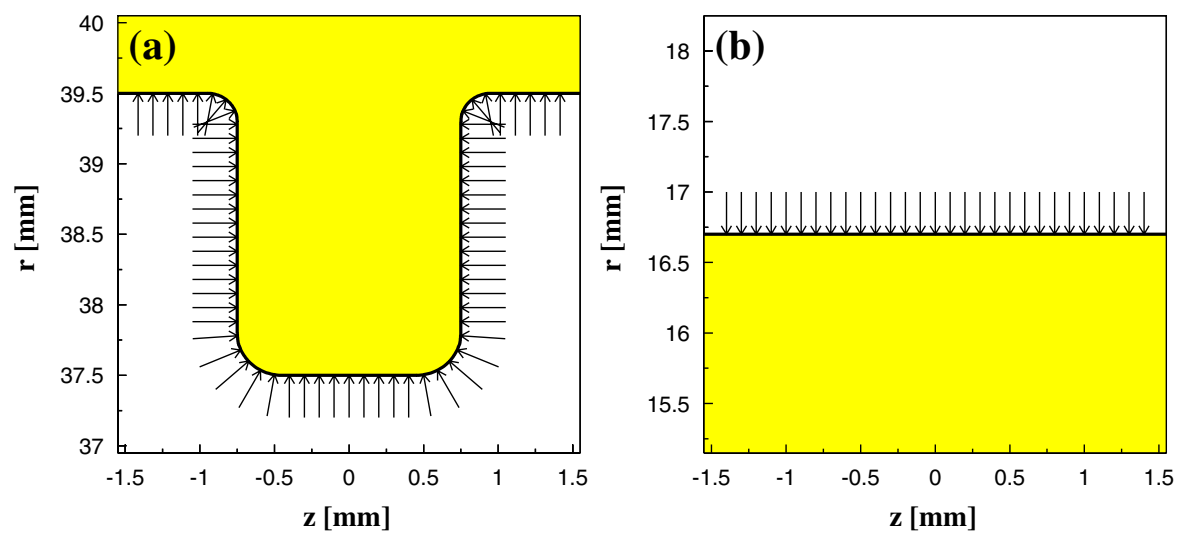

FIG. 4. Initial positions of primary electrons for the (a) outer and (b) inner conductors of the coaxial line are indicated by arrows for a pitch of $3.0 \mathrm{~mm}$ and a depth of $2.0 \mathrm{~mm}$. In this example, $N_{p}^{(z)}=94$ and $\tilde{N}_{p}^{(z)}=58$. 

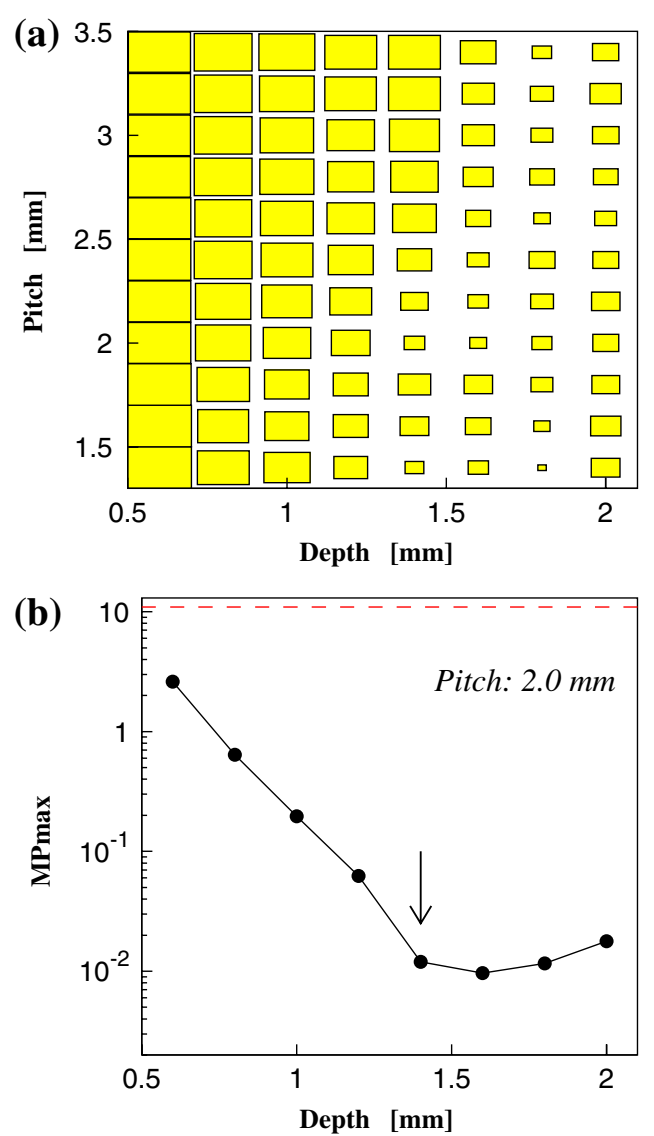

FIG. 5. Maximum values of $\tilde{\mathcal{M}}$ in each multipactoring zone map (a) as functions of pitch and depth, shown as areas of boxes in a log-scale plot, and (b) as a function of depth for a pitch of $2.0 \mathrm{~mm}$, where the vertical arrow indicates the selected value of depth, and the horizontal dashed line is for the ungrooved coaxial line.

and convex regions of the groove structure are equal to half of the pitch $\left(p_{1}=p_{2}=\right.$ pitch $/ 2$ shown in Fig. 2$)$. All the geometries are cylindrically symmetric with respect to the $z$ axis, and the origin of the $z$ axis is defined such that the geometries are symmetric with respect to the $r-\theta$ plane at $z=0$. Figure 3 shows an example of the geometries used in this simulation, which has a pitch of $2.0 \mathrm{~mm}$ and a depth of $1.4 \mathrm{~mm}$; only one-quarter of the full geometry is sufficient due to symmetry.
The electromagnetic field in the grooved coaxial line is not a pure TEM wave, but rather it is a quasi-TEM wave, which is obtained numerically using an electromagneticfield simulator GDFIDL [21], where we set the mesh size to be $0.1 \mathrm{~mm}$. In this simulation, a monochromatic sinusoidal input wave is generated at one port at $z=-52 \mathrm{~mm}$. After the electromagnetic field in the coaxial line becomes steady [22], the field in the region of $-50 \mathrm{~mm}<z<50 \mathrm{~mm}$ at a certain time is stored as $\Re\left(\mathbf{E}_{\mathbf{r}}^{(0)}, \mathbf{E}_{\mathbf{z}}^{(0)}, \mathbf{B}_{\theta}^{(0)}\right)$, and then after a time of $0.25 \times 2 \pi / \omega$, the field in the region of $-50 \mathrm{~mm}<$ $z<50 \mathrm{~mm}$ is stored as $\Im\left(\mathbf{E}_{\mathbf{r}}^{(0)}, \mathbf{E}_{\mathbf{z}}^{(0)}, \mathbf{B}_{\theta}^{(0)}\right)$, where $\omega$ indicates the $\mathrm{rf}$ angular frequency of KEKB $(2 \pi \times$ 508.9 MHz), $\mathbf{E}_{\mathbf{r}}^{(0)}\left(\mathbf{E}_{\mathbf{z}}^{(0)}\right)$ indicates the complex electric field in the $r(z)$ direction, and $\mathbf{B}_{\theta}^{(0)}$ indicates the complex magnetic-flux density in the $\theta$ direction.

The electromagnetic fields inside the grid cells of GDFIDL are calculated by linear interpolation. The exact shapes of the grooved conductor surfaces, including the blended edges and corners, are taken into account in this multipactoring simulation.

\section{B. Modified representation of multipactoring}

The definition of the quantity to represent multipactoring: $\mathcal{M}$, which is described in [18], is modified for nonsmooth grooved surfaces as follows:

$$
\begin{aligned}
\tilde{\mathcal{M}}\left(P_{\mathrm{in}}, \Gamma\right)= & \frac{1}{\tilde{N}_{p}^{(z)} N_{p}^{\left(\phi_{0}^{(\mathrm{I})}\right)} N_{p}^{\left(\phi_{0}^{(\mathrm{R})}\right)}} \sum_{l=1}^{N_{p}^{(z)}} \sum_{k_{i}=1}^{N_{p}^{\left(\phi_{0}^{(\mathrm{I})}\right)}} \sum_{k_{r}=1}^{\left.N_{p}^{(\mathrm{R})}\right)} \\
& \times \frac{r_{l}}{r_{\mathrm{in}}+r_{\text {out }}} f\left[N_{\mathrm{imp}}\left(P_{\mathrm{in}}, \Gamma ; k_{i}, k_{r}, l\right), 10\right],
\end{aligned}
$$

where $N_{p}^{(z)}$ is the number of initial primary electrons uniformly distributed along the conductor surface in the $z$ direction in the region of - pitch $/ 2<z<$ pitch $/ 2$ with a step size of $0.1 \mathrm{~mm}\left(\Delta_{\text {ini }}\right), l$ is an index for the initial position, $k_{i}$ and $k_{r}$ are indices for the initial phases of the input and reflected waves, respectively, $r_{\text {in }}\left(r_{\text {out }}\right)$ is the radius of the inner (outer) conductor: $16.7 \mathrm{~mm}$ $(38.5 \mathrm{~mm}), r_{l}=r_{\text {in }}\left(r_{l}=r_{\text {out }}\right)$ if the initial electron starts from the inner (outer) conductor, $r_{l} /\left(r_{\text {in }}+r_{\text {out }}\right)$ is a normalization factor for the number of initial primary electrons in the $\theta$ direction, the function $f$ is defined as
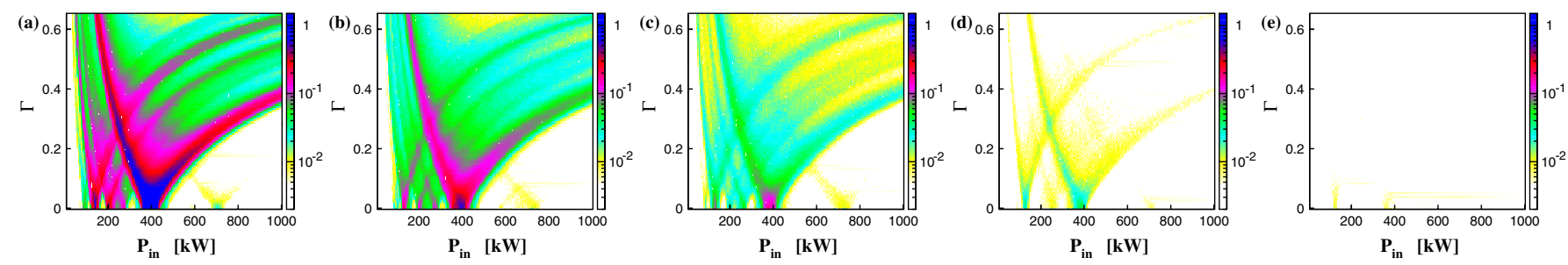

FIG. 6. Multipactoring zone maps for depths of (a) $0.6 \mathrm{~mm}$, (b) $0.8 \mathrm{~mm}$, (c) $1.0 \mathrm{~mm}$, (d) $1.2 \mathrm{~mm}$, and (e) $1.4 \mathrm{~mm}$ and for a pitch of $2.0 \mathrm{~mm}$, where the corresponding MPmax values are shown in Fig. 5(b). 


$$
f(x, a)= \begin{cases}x & (x \geqq a) \\ 0 & (x<a)\end{cases}
$$

and $N_{\text {imp }}$ is the number of impacts of the multipactoring electron as defined in Sec. II A of [18] except that the $z$ region for this multipactoring simulation is $-50 \mathrm{~mm}<$ $z<50 \mathrm{~mm}$ instead of $-200 \mathrm{~mm}<z<-50 \mathrm{~mm}$.

A primary electron is placed on the conductor surface with an initial energy of zero at a specified position, and its motion is computed according to the relativistic equation
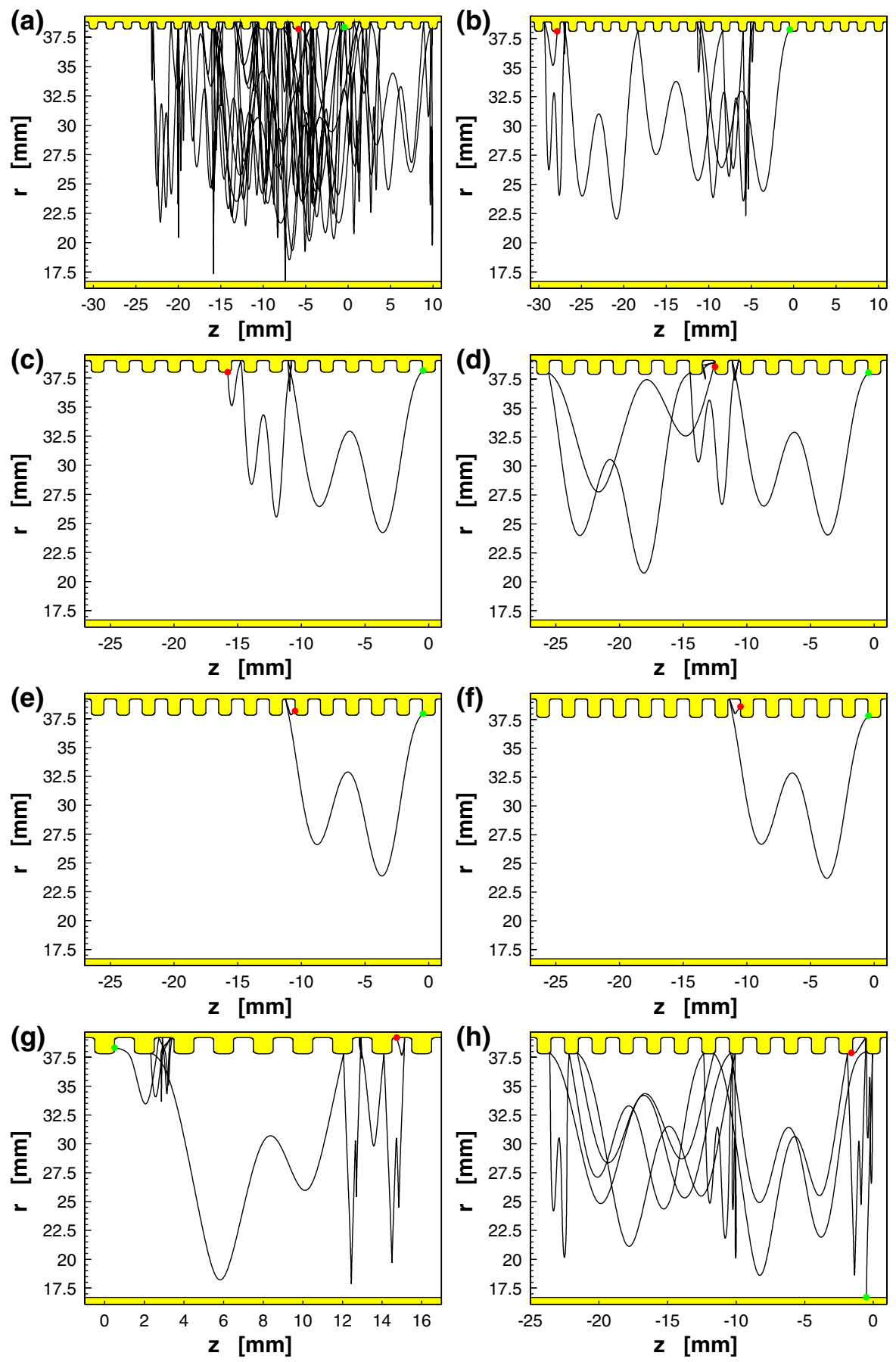

FIG. 7. Examples of multipactoring-electron trajectories in the case of $P_{\text {in }}=390 \mathrm{~kW}, \Gamma=0$, and a pitch of $2.0 \mathrm{~mm}$. For depths: (a) $0.6 \mathrm{~mm}$, (b) $0.8 \mathrm{~mm}$, (c) $1.0 \mathrm{~mm}$, (d) $1.2 \mathrm{~mm}$, (e) $1.4 \mathrm{~mm}$, and (f) $1.6 \mathrm{~mm}$ for $\phi_{0}^{(\mathrm{I})}=4.398$ rad and the same initial position. For (g) $\phi_{0}^{(\mathrm{I})}=4.021 \mathrm{rad}$ and $(\mathrm{h}) \phi_{0}^{(\mathrm{I})}=1.885 \mathrm{rad}$ for a depth of $1.4 \mathrm{~mm}$. The green dots indicate the starting points, and the red dots indicate the ending points, where all the impact energies at the last impact in these examples are lower than $E_{\min }=100 \mathrm{eV}$. Defining a multipactoring order $(\eta)$ as (simulated time) $\times \omega /(2 \pi) / N_{\text {imp }}$, the $\eta\left(N_{\text {imp }}\right)$ values are $1.60(65), 1.48(12), 1.16(5), 1.49(7), 1.31(2)$, 1.38 (2), 1.26 (11), and 1.77 (13) for (a), (b), (c), (d), (e), (f), (g), and (h), respectively. 
of motion. When the electron impacts the inner or outer conductor, the impact energy $\left(E_{\mathrm{imp}}\right)$ is compared with the specified minimum $\left(E_{\min }=100 \mathrm{eV}\right)$ and maximum $\left(E_{\max }=1200 \mathrm{eV}\right)$ energies, where the value of the secondary electron yield $(\delta)$ of the copper conductor is not considered; instead, the range for $\delta>1$ is considered in setting $E_{\min }$ and $E_{\max }$. If the impact energy lies in the range of $E_{\text {min }}<E_{\text {imp }}<E_{\max }$, a secondary electron is launched, from the position where the primary one impacted the conductor, with an initial energy of $5 \mathrm{eV}$ in the direction perpendicular to the conductor surface. The same procedure is then repeated by regarding the secondary electron
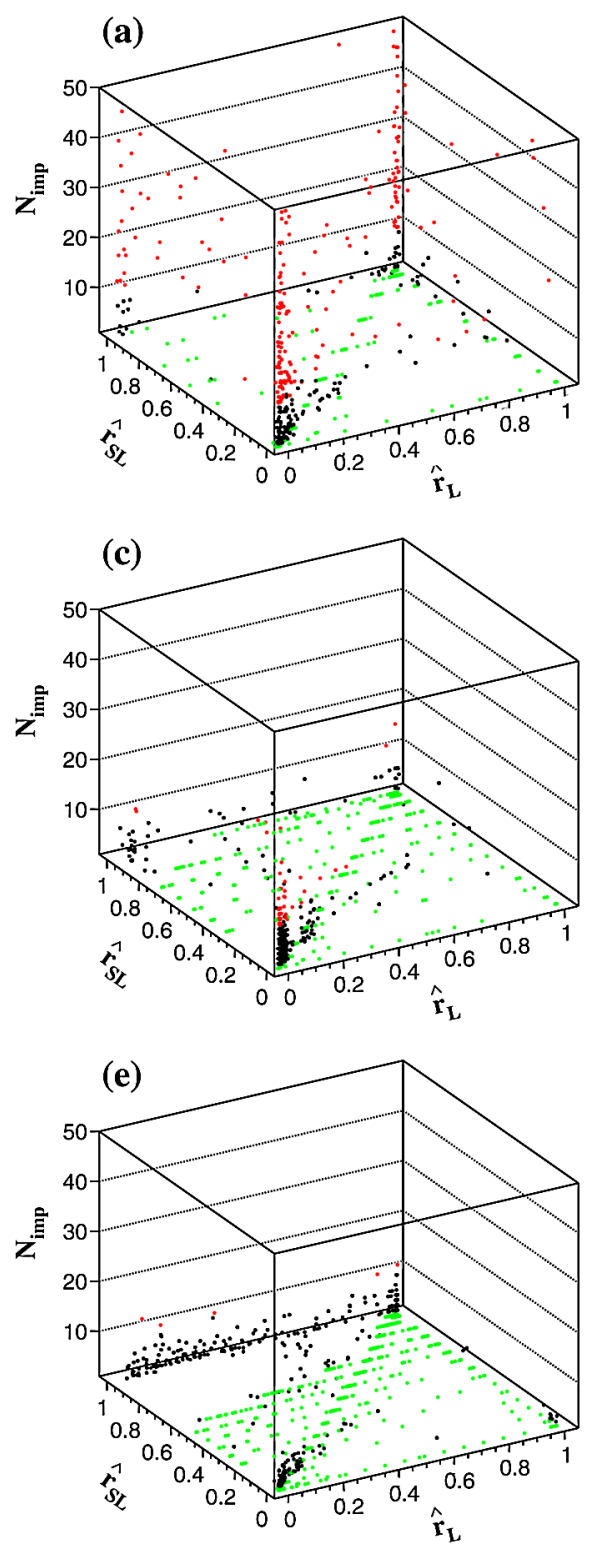

as the primary electron until one of the following four conditions is satisfied: (i) the impact energy is outside the range, $E_{\mathrm{imp}}<E_{\min }$ or $E_{\mathrm{imp}}>E_{\max }$; (ii) the electron is accelerated into the conductor in the first step immediately after it was placed on, or launched from, the conductor; (iii) the multipactoring electron leaves the groove structure region (i.e. $z<-50 \mathrm{~mm}$ or $z>50 \mathrm{~mm}$ ); (iv) the number of impacts exceeds a specified number: $N_{\max }=100 . N_{\text {imp }}$ in Eq. (1) is the number of impacts of the above multipactoring electron for each set of $\left(P_{\mathrm{in}}, \Gamma ; k_{i}, k_{r}, l\right)$.

One significant difference from the definition in [18] is the normalization factor for the initial positions of the
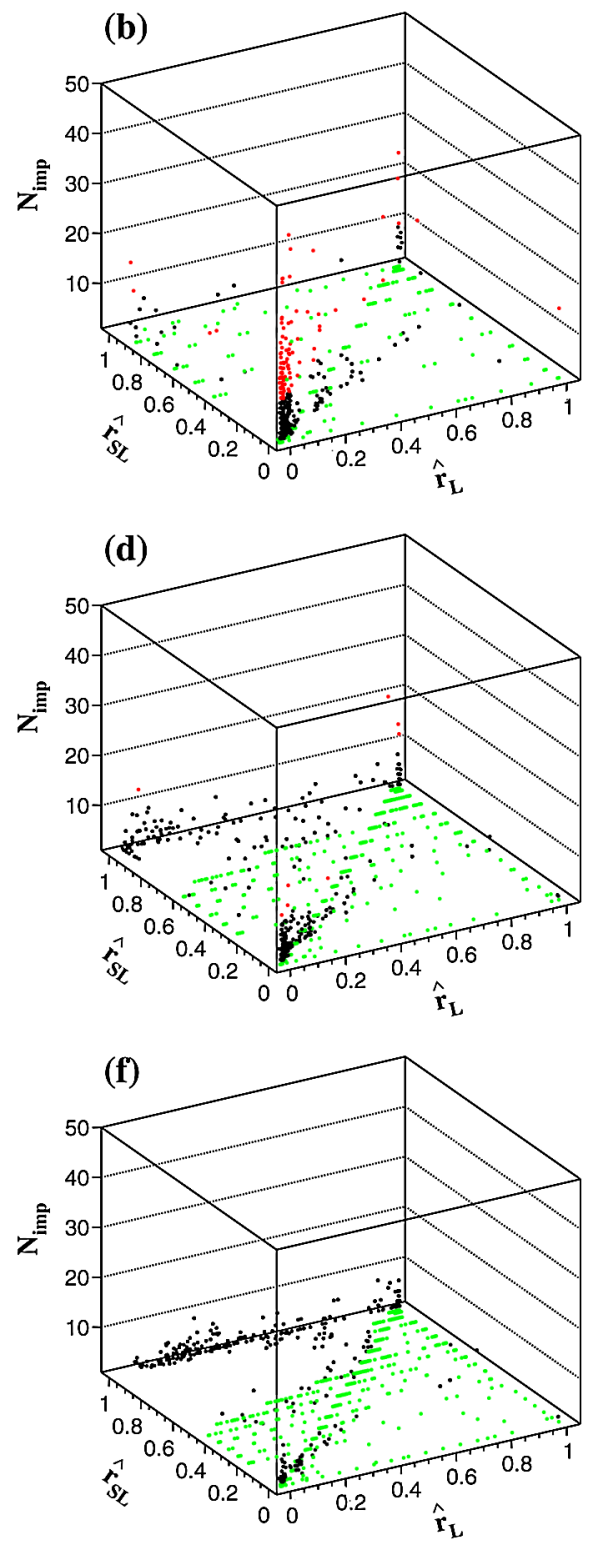

FIG. 8. Number of impacts as functions of the normalized radial positions at the second last and the last impacts of electrons for depths: (a) $0.6 \mathrm{~mm}$, (b) $0.8 \mathrm{~mm}$, (c) $1.0 \mathrm{~mm}$, (d) $1.2 \mathrm{~mm}$, (e) $1.4 \mathrm{~mm}$, and (f) $1.6 \mathrm{~mm}$ for a pitch of $2.0 \mathrm{~mm}, P_{\text {in }}=390 \mathrm{~kW}$, and $\Gamma=0$. Different points correspond to different initial rf phases and electron positions. The region of $\hat{r}=0(\hat{r}=1)$ corresponds to the radial position of $r_{\text {out }}-$ depth $/ 2\left(r_{\text {out }}+\right.$ depth/2). In the case of $N_{\text {imp }}=1$, shown in green, $\hat{r}_{\mathrm{SL}}$ indicates the initial position. Electrons with $N_{\text {imp }}$ in the range of $1<N_{\text {imp }}<10\left(10 \leqq N_{\text {imp }} \leqq 50\right)$ are shown in black (red). The electrons for $N_{\text {imp }}>50$ are not shown here. 
primary electrons in the $z$ direction, which is $\tilde{N}_{p}^{(z)}$ defined as $\left[\operatorname{int}\left(\operatorname{pitch} / \Delta_{\text {ini }}\right)-1\right] \times 2$. The equality $\tilde{N}_{p}^{(z)}=N_{p}^{(z)}$ holds for ungrooved coaxial lines. Figure 4 shows an example of the initial positions of primary electrons for a pitch of $3.0 \mathrm{~mm}$ and a depth of $2.0 \mathrm{~mm}$. Another significant difference is the two independent initial-phase parameters: $\phi_{0}^{(\mathrm{I})}=2 \pi \times\left(k_{i}-1\right) / N_{p}^{\left(\phi_{0}^{(\mathrm{I})}\right)} \quad$ and $\quad \phi_{0}^{(\mathrm{R})}=2 \pi \times\left(k_{r}-\right.$ $1) / N_{p}^{\left(\phi_{0}^{(\mathrm{R})}\right)}$, which are used in Eqs. (3)-(5), where $N_{p}^{\left(\phi_{0}^{(\mathrm{I})}\right)}$ and $N_{p}^{\left(\phi_{0}^{(\mathrm{R})}\right)}$ are the numbers of sampling points uniformly distributed in the region of $[0,2 \pi)$ for the input and reflected waves, respectively; we set $N_{p}^{\left(\phi_{0}^{(\mathrm{I})}\right)}=N_{p}^{\left(\phi_{0}^{(\mathrm{R})}\right)}=50 \mathrm{in}$ this simulation.

\section{Superposition of input and reflected waves}

With the complex electromagnetic field of the quasiTEM wave obtained using GDFIDL, $\left(\mathbf{E}_{\mathbf{r}}^{(0)}, \mathbf{E}_{\mathbf{z}}^{(0)}, \mathbf{B}_{\theta}^{(0)}\right)(r, z)$, the real electromagnetic field at the space-time $(r, z, t)$ for the multipactoring simulation is calculated as

$$
\begin{aligned}
E_{r}(r, z, t)= & \Re\left\{\mathbf{E}_{\mathbf{r}}^{(0)}(r, z) e^{-i\left(\omega t+\phi_{0}^{(\mathrm{I})}\right)}\right. \\
& \left.+\Gamma \mathbf{E}_{\mathbf{r}}^{(0)}(r,-z) e^{-i\left(\omega t+\phi_{0}^{(\mathrm{R})}\right)}\right\}, \\
E_{z}(r, z, t)= & \Re\left\{\mathbf{E}_{\mathbf{z}}^{(0)}(r, z) e^{-i\left(\omega t+\phi_{0}^{(\mathrm{I})}\right)}\right. \\
+ & \left.\Gamma\left[-\mathbf{E}_{\mathbf{z}}^{(0)}(r,-z) e^{-i\left(\omega t+\phi_{0}^{(\mathrm{R})}\right)}\right]\right\}, \\
B_{\theta}(r, z, t)= & \Re\left\{\mathbf{B}_{\theta}^{(0)}(r, z) e^{-i\left(\omega t+\phi_{0}^{(\mathrm{I})}\right)}\right. \\
& \left.+\Gamma\left[-\mathbf{B}_{\theta}^{(0)}(r,-z) e^{-i\left(\omega t+\phi_{0}^{(\mathrm{R})}\right)}\right]\right\}, \\
E_{\theta} & =B_{r}=B_{z}=0,
\end{aligned}
$$

where $\left(E_{r}, E_{\theta}, E_{z}\right)$ and $\left(B_{r}, B_{\theta}, B_{z}\right)$ indicate the electric and magnetic-flux vectors, respectively, $\Gamma$ is the real reflection coefficient, and the reflected waves are calculated as mathematical space reflections of the input waves with respect to the $r-\theta$ plane at $z=0$. Because $\phi_{0}^{(\mathrm{R})}$ varies from 0 to $2 \pi$ in the summation in Eq. (1), multipactoring zone maps in this simulation are symmetric between the overcoupling $(\Gamma>0)$ and undercoupling $(\Gamma<0)$ regions, so that we calculate Eq. (1) only for the overcoupling and optimum-coupling regions $(\Gamma \geqq 0)$.

\section{Selection of pitch and depth values}

Multipactoring zone maps, which show the quantity $\tilde{\mathcal{M}}$ defined in Eq. (1), have been computed for pitches from 1.4 to $3.4 \mathrm{~mm}$ and depths from 0.6 to $2.0 \mathrm{~mm}$ with a step size of $0.2 \mathrm{~mm}$, giving a total of 88 maps [23]. In each map, the input rf power range is set to be $10 \mathrm{~kW}$ to $1 \mathrm{MW}$, and the $\Gamma$ range is set to be 0 to 0.65 . Multipactoring suppression effects can be estimated by taking the maximum value of $\tilde{\mathcal{M}}$ in each map (MPmax). Figure 5(a) shows the MPmax values as functions of pitch and depth. Since depths should be small due to the presence of cooling channels in the outer conductor, and pitches smaller than $2.0 \mathrm{~mm}$ are technically difficult in machining, we have selected a pitch of $2.0 \mathrm{~mm}$ and a depth of $1.4 \mathrm{~mm}$, although there is a minimum at a pitch of $1.4 \mathrm{~mm}$ and a depth of $1.8 \mathrm{~mm}$, as seen in Fig. 5(a). Figure 5(b) shows MPmax as a function of depth for a pitch of $2.0 \mathrm{~mm}$, where the corresponding multipactoring zone maps are shown in Figs. 6(a)-6(e).

Figures 7(a)-7(f) show an illustration of multipactoring suppression for $P_{\text {in }}=390 \mathrm{~kW}$ and $\Gamma=0$ with a pitch of $2.0 \mathrm{~mm}$ and various depths. For a depth of $0.6 \mathrm{~mm}$ in Fig. 7 (a), the electron undergoes multipactoring until the 65th impact with an impact energy of $4.4 \mathrm{eV}$, which is lower than $E_{\min }=100 \mathrm{eV}$. For a depth of $0.8 \mathrm{~mm}$ in Fig. 7(b), the electron undergoes multipactoring, however, it finishes earlier since a deeper groove structure is expected to reduce the stability of multipactoring. For depths of 1.0 and $1.2 \mathrm{~mm}$ in Figs. 7(c) and 7(d), respectively, the electron undergoes multipactoring with only several impacts; in this case, more impacts for the deeper grooves. Further suppression is obtained for depths of 1.4 and $1.6 \mathrm{~mm}$ in Figs. 7 (e) and 7(f), respectively, where the initial electron impacts on the concave region of the groove, and subsequent secondary electrons do not obtain sufficient energy from the low electric field in the concave region. This effect can be statistically seen in Figs. 8(a)-8(f), which show the number of impacts as functions of the normalized radial positions, defined as

$$
\hat{r}=\left[r-\left(r_{\text {out }}-\operatorname{depth} / 2\right)\right] / \text { depth, }
$$

at the last impact $\left(\hat{r}_{\mathrm{L}}\right)$ and the second last impact $\left(\hat{r}_{\mathrm{SL}}\right)$. A clear cluster appears in the range of $\hat{r}_{\mathrm{SL}}>0.9$ for depths of 1.4 and $1.6 \mathrm{~mm}$ [see Figs. 8(e) and 8(f), respectively], where most of the electrons undergo only a few impacts. Actually, all of the electrons in the range of $\hat{r}_{\mathrm{SL}}>0.9$ for a depth of $1.4 \mathrm{~mm}(1.6 \mathrm{~mm})$ move only in the region of $-0.3<\hat{r} \leqq 1.0(-0.2<\hat{r} \leqq 1.0)$, which is very close to the outer conductor, between the second last and the last

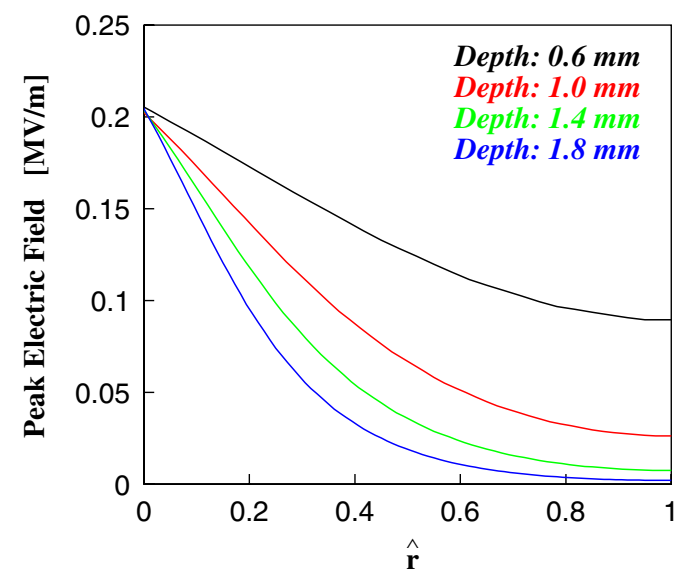

FIG. 9. Peak electric field at $z=1.0 \mathrm{~mm}$ as a function of $\hat{r}$ for a pitch of $2.0 \mathrm{~mm}$ and various depths for an input $\mathrm{rf}$ power of $1 \mathrm{MW}(\mathrm{CW})$, obtained by the simulation using GDFIDL. 


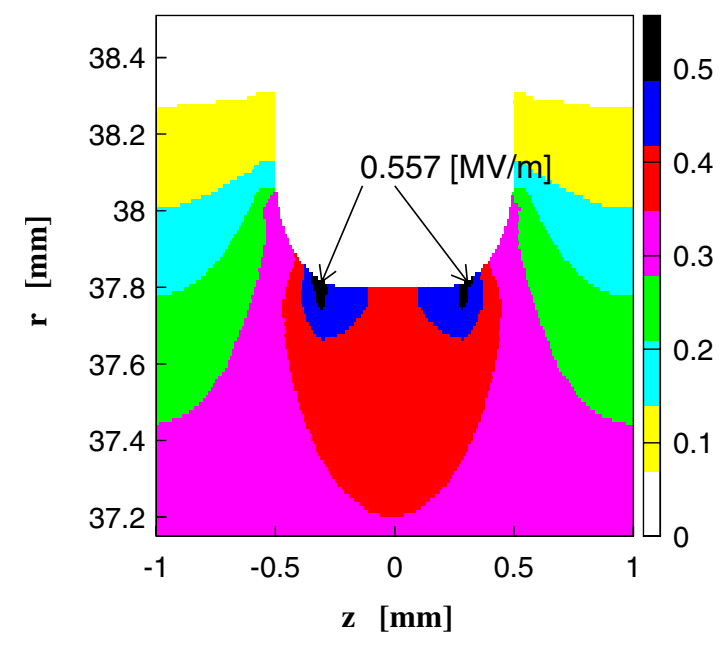

FIG. 10. Peak electric field (in $\mathrm{MV} / \mathrm{m}$ ) as functions of $z$ and $r$ around the groove for a pitch of $2.0 \mathrm{~mm}$ and a depth of $1.4 \mathrm{~mm}$ for an input rf power of $1 \mathrm{MW}(\mathrm{CW})$, obtained by the simulation using GDFIDL. The maximum field value is $0.557 \mathrm{MV} / \mathrm{m}$ near the edges.
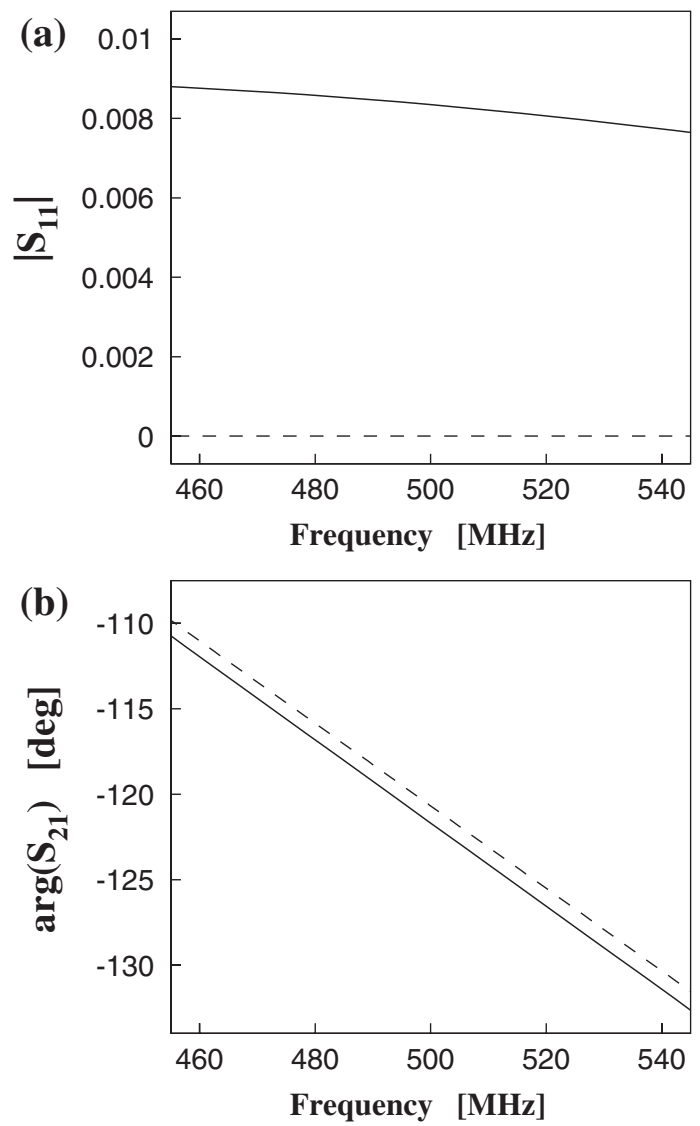

FIG. 11. (a) $\left|S_{11}\right|$ and (b) $\arg \left(S_{21}\right)$ of the $S$ parameters for the grooved coaxial line with a pitch of $2.0 \mathrm{~mm}$ and a depth of $1.4 \mathrm{~mm}$ (solid lines). The dashed lines indicate the $S$ parameters for the ungrooved coaxial line. impact, and all the multipactorings for the range of $\hat{r}_{\mathrm{SL}}>$ 0.9 terminate because the impact energies are lower than $E_{\min }=100 \mathrm{eV}$ at the last impact. This effect is attributed to the lower electric field in the concave region of deeper grooves, as shown in Fig. 9.

Figures $7(\mathrm{~g})$ and $7(\mathrm{~h})$ show examples of surviving multipactoring electrons for the selected pitch and depth in the case of $P_{\text {in }}=390 \mathrm{~kW}$ and $\Gamma=0$. It should be noted that multipactoring with $N_{\text {imp }} \geqq 10$ for the selected pitch and

(a)

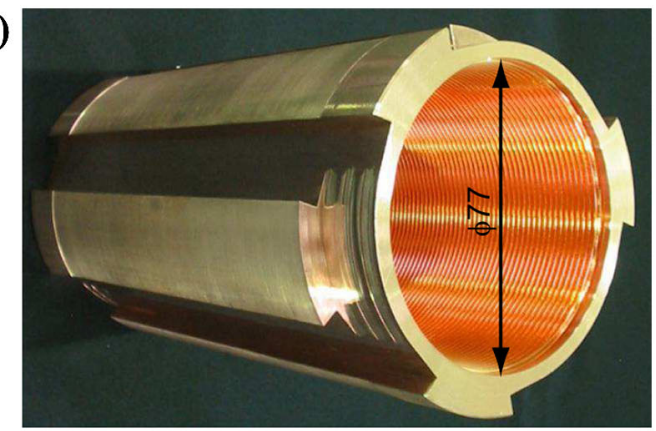

(b)

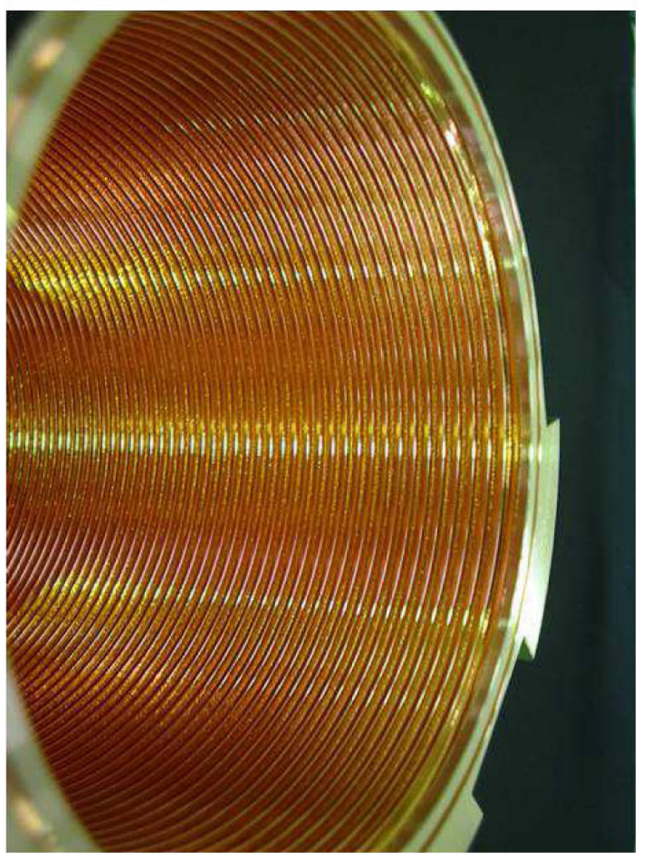

FIG. 12. (a) Grooved outer conductor of the coaxial line, made of oxygen-free copper, for the first grooved coupler, where the dimension is given in millimeters. (b) Magnification of (a).

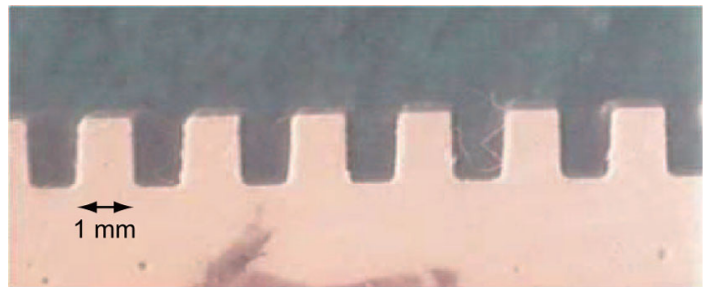

FIG. 13. Replica of the grooves for the first grooved coupler shown in Fig. 12, which is made of silicone. 
depth can occur with an initial rf phase only in a limited region, and that it terminates much earlier than for the shallower grooves.

We have checked the maximum peak electric field for $1 \mathrm{MW}$ input rf power (CW) using GDFIDL, which is computed to be $0.557 \mathrm{MV} / \mathrm{m}$ as shown in Fig. 10 for the selected pitch and depth. This field value is lower than that on the inner conductor of the ungrooved coaxial line $(0.717 \mathrm{MV} / \mathrm{m})$.

We have also checked if the groove structure with the selected pitch and depth significantly affects the rf properties of the cavity measured through the grooved coupler, by using GDFIDL with the same mesh size of $0.1 \mathrm{~mm}$, where the length of the groove structure along the coaxial line was extended to $180 \mathrm{~mm}$ adopted for the actual grooved couplers. In this simulation, a broadband input wave with a

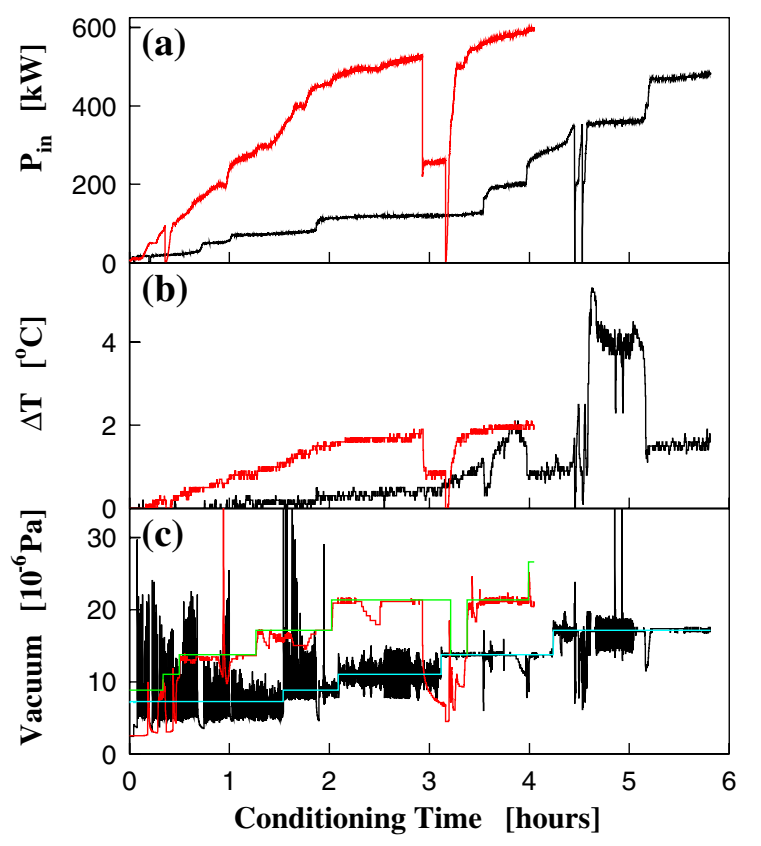

FIG. 14. Conditioning histories of an ungrooved coupler (black) and the first grooved coupler (red), where a conditioning time of 0 indicates the start of the first conditioning for both couplers, on the (a) input rf power, (b) temperature difference of the cooling water between into and out of the outer conductor of the coaxial line, and (c) vacuum pressure. The green (light blue) line in (c) indicates the reference vacuum pressure specified in the automatic conditioning of the grooved (ungrooved) coupler by computer control. If the vacuum pressure is higher than the reference, the power is slightly stepped down until the vacuum pressure becomes lower than the reference, and then the power is slightly stepped up as long as the vacuum pressure is lower than the reference. After keeping the input rf power with the same reference, the vacuum pressure gradually decreases, and more power can be input. This process is known as "conditioning," where gradual increases of the reference are required at higher input rf powers due to the greater rf surface heating. The sudden power drop about three hours after the start of conditioning for the grooved coupler was caused by the klystron overdrive. center frequency of $500 \mathrm{MHz}$ and a bandwidth of $100 \mathrm{MHz}$ is generated in the time domain at one port at $z=$ $-100.5 \mathrm{~mm}$ (port 1), and detected at the another port at $z=100.5 \mathrm{~mm}$ (port 2). The time-dependent signal at port 2 is stored until all the signal heights of the port modes decay to $10^{-9}$ times the signal height of the input wave, then Fourier transformed to obtain $S$ parameters. The simulated time, from the input-wave excitation to the signal-height decay to the above-mentioned level, is $117 \mathrm{~ns}$, so that there is no significant fine structure in an interval of $1 /(117 \mathrm{~ns}) \approx 8.5 \mathrm{MHz}$ in the frequency domain, which is much wider than typical widths of the resonator impedance of the accelerating mode in the ARES cavity: $\approx 10 \mathrm{kHz}$. Figure 11 (a) shows the simulation result
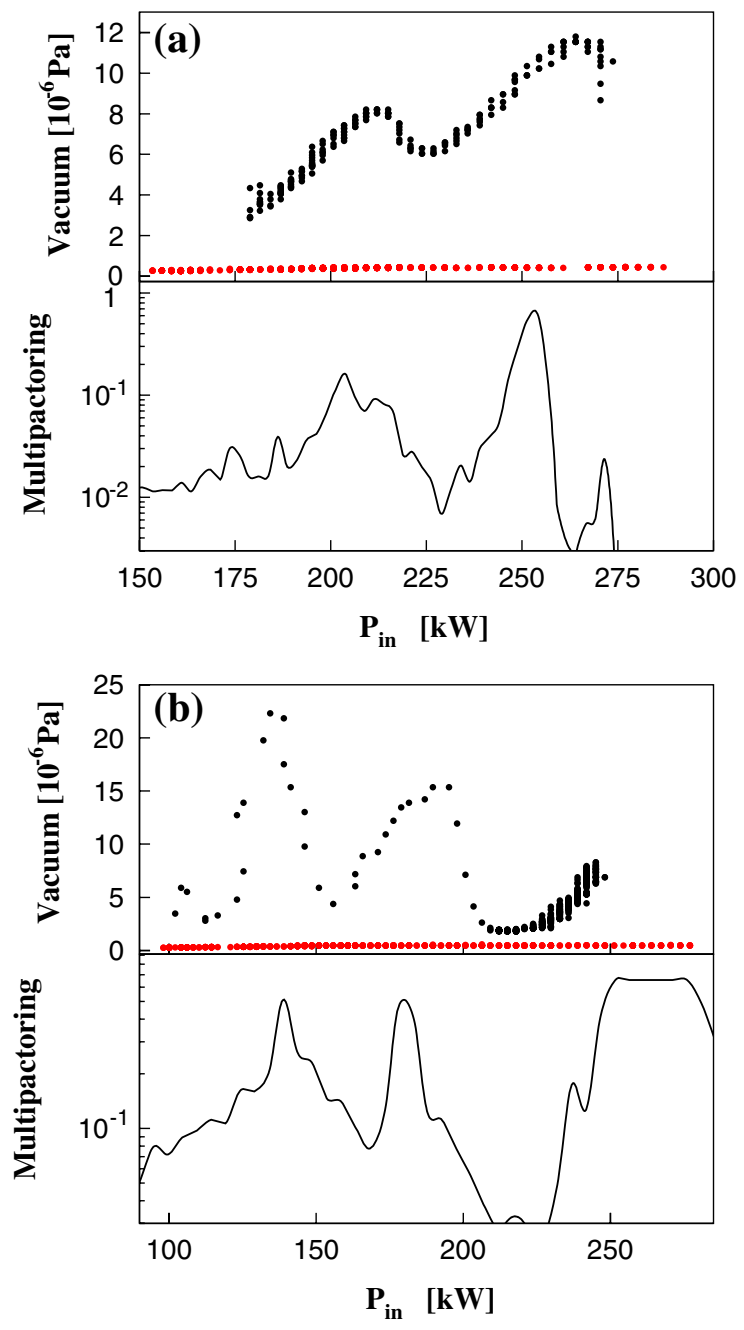

FIG. 15. Comparisons of the vacuum data on CavityM (upper) with the calculation results for $\mathcal{M}$ (lower) as a function of the input rf power for the operating points of CavityM (a) in the overcoupling, (b) optimum-coupling and undercoupling regions. The black (red) dots indicate measurements of the vacuum pressure without (with) grooves. The trajectories of the operating points for the black and red dots in (a) and the black and red dots in (b) are shown in Figs. 16(a)-16(d), respectively. 

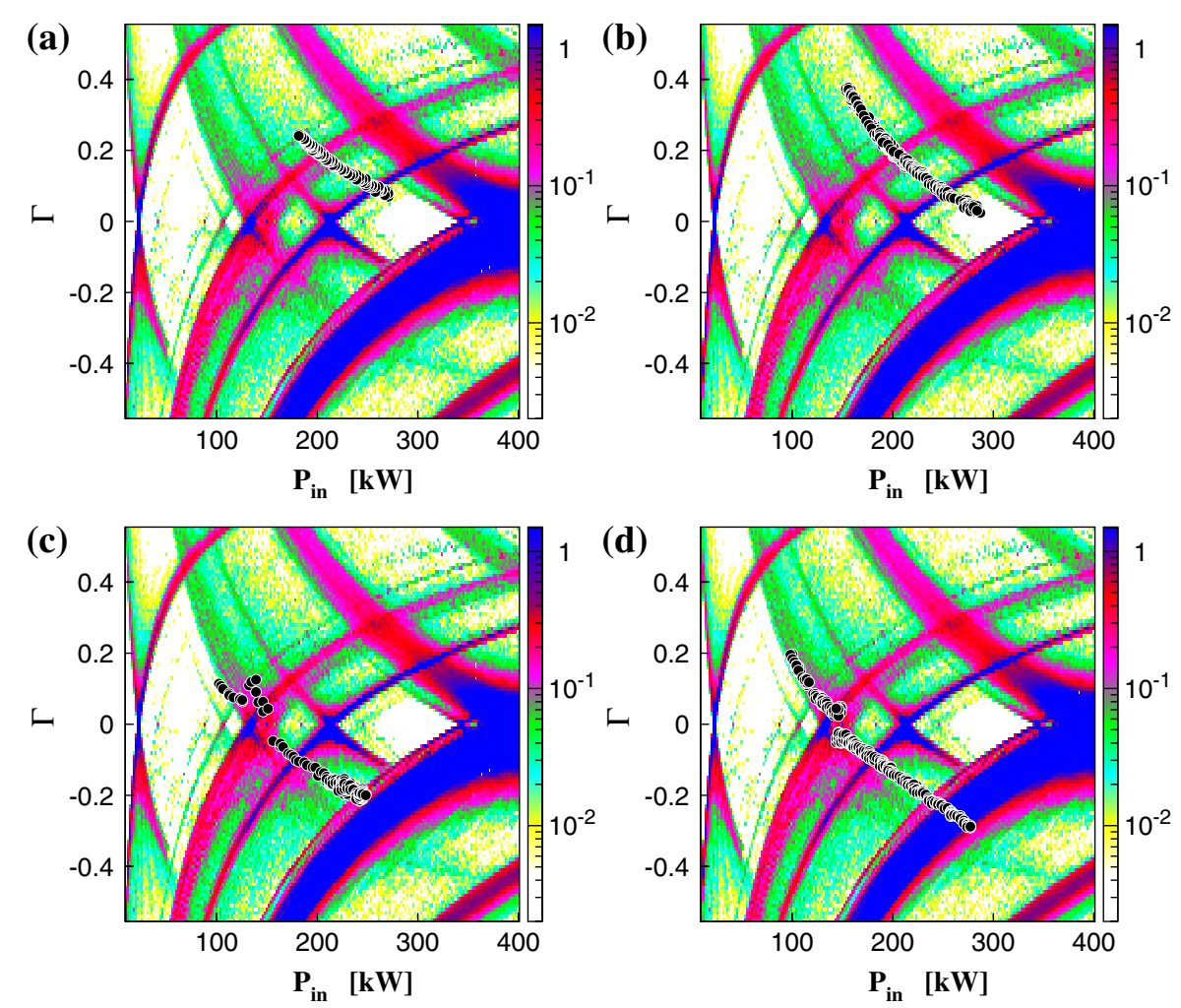

FIG. 16. Trajectories of the operating point of CavityM, superimposed on the multipactoring zone map for the ARES cavity with the ungrooved coupler: $\mathcal{M}$ from [18]. The map is asymmetric between the overcoupling and undercoupling regions. The black circles indicate measurements of the input rf power and the reflection coefficient (a) in the overcoupling region without grooves on the outer conductor of the coaxial line, (b) in the overcoupling region with grooves, (c) in the optimum-coupling and undercoupling regions without grooves, and (d) in the optimum-coupling and undercoupling regions with grooves.

for the absolute value of the reflection coefficient $\left(S_{11}\right)$, demonstrating that the reflected power is very small. Figure 11(b) shows the simulation result for the argument of the transmission coefficient $\left(S_{21}\right)$, demonstrating that the phase shift from the ungrooved coaxial line is small and nondispersive. Therefore, the groove structure has a negligible effect on the rf properties of the cavity around an $\mathrm{rf}$ frequency of 508.9 MHz.

\section{COUPLER PRODUCTION}

Fine grooves were formed in a coaxial line using a metal slitting saw. The edges were then blended using a diamond bit. Finally, electropolishing was applied to the inner surface of the coaxial line to completely remove burrs. Figure 12 shows photographs of the grooved coaxial line for the first grooved coupler. We checked the dimension errors of the grooves by making a replica, as shown in Fig. 13.

\section{DEMONSTRATIONS}

\section{A. High-power tests}

Figure 14 shows the conditioning history for the first grooved coupler tested in a test stand together with an example of ungrooved couplers, where the vacuum pressures were kept below the specified levels, the green line for the grooved coupler and the light blue line for the ungrooved coupler in Fig. 14(c), by computer control, and the reflected power was set to be almost zero $(\Gamma \cong 0)$ by adjusting the coupling factor of the output coupler [15]. Clear power-dependent temperature rises on the outer conductor of the ungrooved coupler are observed around input rf powers of 200 and $350 \mathrm{~kW}$ [see the black lines in Figs. 14(a) and 14(b)], which can be identified with heat input to the outer conductor by multipactoring because clear discharges in the coaxial line were also observed during those temperature rises. Those power levels for the temperature rises are in good agreement with the multipactoring zones predicted by the simulation: 200 to $230 \mathrm{~kW}$ and 350 to $440 \mathrm{~kW}$ for $\Gamma=0$, as shown in Fig. 6 (a). In contrast, no power-dependent temperature rise or discharge was observed for the grooved coupler.

So far, we have made five grooved couplers, and tested them in the same test stand for input rf powers up to or over $400 \mathrm{~kW}$. None of them exhibited any multipactoring problems on the coaxial line.

\section{B. KEKB operations}

We installed one of the grooved couplers to CavityM used in KEKB operations. Figure 15(a) shows measurements 
of the vacuum pressures in CavityM with an ungrooved coupler (black dots) and this grooved coupler (red dots) for almost the same operational routes in the overcoupling region as those shown in Figs. 16(a) and 16(b), respectively, together with the corresponding calculation results for $\mathcal{M}$. For the ungrooved coupler, there are powerdependent pressure rises as predicted by the multipactoring simulation, as shown in Fig. 15(a) (black dots and solid line). In contrast, no power-dependent pressure rises are observable in Fig. 15(a) (red dots). Figure 15(b) shows the same kind of comparison for the optimum-coupling and undercoupling regions for the ungrooved (black dots) and grooved (red dots) couplers for almost the same operational routes as those shown in Figs. 16(c) and 16(d), respectively. The grooved coupler installed to CavityM was used without any problems for over 100 days of KEKB operation.

\section{CONCLUSIONS}

We have overcome the multipactoring problem of CavityM on the coaxial line of the couplers by fine grooving of the conductor surfaces, where this approach is based on the multipactoring zone map. The performance of the grooved couplers has been demonstrated in both the highpower tests and KEKB operations.

Most of the ungrooved couplers did not exhibit multipactoring problems in either the high-power tests or during KEKB operations. Thus, in combination with the method described in [18], we can use a mixture of ungrooved and grooved couplers, making it unnecessary to replace all the couplers at once. This means that, when a cavity with an ungrooved coupler starts to exhibit multipactoring in the coaxial line, the operating point of the cavity can be kept in a multipactoring-free region to prevent the accelerator performance from being degraded, and the ungrooved coupler can then be replaced by a grooved one at the earliest opportunity.

\section{ACKNOWLEDGMENTS}

The authors are very grateful to Professor K. Akai, Professor K. Oide, and the other members of the KEKB group for fruitful comments and various support.

[1] T. Kageyama et al., 1st Asian Particle Accelerator Conference (APAC 98), Tsukuba, Japan, 1998 (KEK Report No. KEK-PREPRINT-98-45).

[2] Y. Yamazaki and T. Kageyama, Part. Accel. 44, 107 (1994).

[3] K. Akai et al., Nucl. Instrum. Methods Phys. Res., Sect. A 499, 45 (2003).
[4] S. Kurokawa, Nucl. Instrum. Methods Phys. Res., Sect. A 499, 1 (2003).

[5] M. Kikuchi et al., Nucl. Instrum. Methods Phys. Res., Sect. A 499, 8 (2003).

[6] K. Egawa et al., Nucl. Instrum. Methods Phys. Res., Sect. A 499, 24 (2003).

[7] K. Kanazawa et al., Nucl. Instrum. Methods Phys. Res., Sect. A 499, 66 (2003).

[8] K. Kanazawa et al., Nucl. Instrum. Methods Phys. Res., Sect. A 499, 75 (2003).

[9] M. Arinaga et al., Nucl. Instrum. Methods Phys. Res., Sect. A 499, 100 (2003).

[10] N. Akasaka et al., Nucl. Instrum. Methods Phys. Res., Sect. A 499, 138 (2003).

[11] I. Abe et al., Nucl. Instrum. Methods Phys. Res., Sect. A 499, 167 (2003).

[12] K. Akai et al., Nucl. Instrum. Methods Phys. Res., Sect. A 499, 191 (2003).

[13] The ARES system consists of three cavities, each of which is for acceleration, energy storage, or coupling between the other two. The rf power is fed into the energy-storage cavity, which is a large cylinder in shape, and has a role of an electromagnetic flywheel to stabilize the accelerating mode against beam loading.

[14] F. Naito et al., 1st Asian Particle Accelerator Conference (APAC 98), Tsukuba, Japan, 1998 (KEK Report No. KEK-PREPRINT-98-44).

[15] H. Sakai et al., in Proceedings of the 21st Particle Accelerator Conference, Knoxville, 2005 (IEEE, Piscataway, NJ, 2005), TPPT012.

[16] Multipactoring is a phenomenon arising from secondary electron emissions after impact of primary electrons and repetition of this process driven by the rf field, also known as multipacting.

[17] CavityM is downstream one of the two ARES cavities which were installed in the rf station called D4C in KEKB.

[18] T. Abe et al., Phys. Rev. ST Accel. Beams 9, 062002 (2006).

[19] H. Padamsee, D. Proch, P. Kneisel, and J. Mioduszewski, IEEE Trans. Magn. 17, 947 (1981).

[20] R. L. Geng, H. Padamsee, S. Belomestnykh, P. Goudket, D. M. Dykes, and R. G. Carter, Nucl. Instrum. Methods Phys. Res., Sect. A 508, 227 (2003).

[21] http://www.gdfidl.de/.

[22] The condition for steady state in this simulation is that the port signal at the other port at $z=52 \mathrm{~mm}$ is equal to $A \times$ $\sin \left[\omega\left(t-t_{0}\right)\right]$ to an accuracy of $0.3 \%$, where $A$ is the last maximum signal height, and $t_{0}$ is the time of the last zero crossing.

[23] Typical computation times to obtain each map using recent 64-bit processors with a clock frequency of $2.7 \mathrm{GHz}$ are two days for the parallel computation of the electromagnetic field by GDFIDL using eight processor cores, and two weeks for the multipactoring simulation in serial computing. All the computation tasks to obtain the 88 maps were distributed among the 256 processor cores in the PC cluster. 